\title{
A Statistical Method for Footprints Analysis based on Large-scale High-density Piezoresistive Films
}

\author{
Bo Wang \\ Shenzhen Institutes of Advanced \\ Technology, Chinese Academy of \\ Sciences, Shenzhen, China \\ bo.wang1@siat.ac.cn
}

\author{
Chengxiang Liu \\ Shenzhen Institutes of Advanced \\ Technology, Chinese Academy of \\ Sciences, Shenzhen, China \\ cx.liu@siat.ac.cn
}

\author{
Peng Shang* \\ Shenzhen Institutes of Advanced \\ Technology, Chinese Academy of \\ Sciences, Shenzhen, China \\ peng.shang@siat.ac.cn
}

\begin{abstract}
Footprints can provide much valuable information for fall prediction, the diagnosis of many diseases and rehabilitation therapy. This paper aims to propose a statistical method for footprints analysis based on a large-scale high-density piezoresistive film to replace the manual work of obtaining the main parameters of footprint data. Firstly, data acquisition devices are developed to obtain the plantar pressure distribution by measuring the voltage changes caused by the applied pressure on the piezoresistive films. Subsequently, a specific software is developed to receive the data from the designed signal acquisition devices through the serial port and visualize the footprints, and a statistical method is proposed to distinguish between left footprint and right footprint and to obtain the direction of the footprints and the step length. Eventually, a series of experiments are conducted to obtain the accuracy of the proposed method used for different people's feet and with different walking speeds.
\end{abstract}

\section{CCS CONCEPTS}

- Applied computing; • Life and medical sciences; • Health care information systems;

\section{KEYWORDS}

Statistical method, footprints analysis, piezoresistive films

\section{ACM Reference Format:}

Bo Wang, Chengxiang Liu, and Peng Shang*. 2021. A Statistical Method for Footprints Analysis based on Large-scale High-density Piezoresistive Films. In 2021 International Symposium on Biomedical Engineering and Computational Biology (BECB 2021), August 13-15, 2021, Nanchang, China. ACM, New York, NY, USA, 5 pages. https://doi.org/10.1145/3502060.3502148

\section{INTRODUCTION}

Many foot pathologies can be diagnosed by the footprints, such as the type of foot (hallux valgus, clubfoot, and pes planus) [1], diabetic foot [2], pathological plantar hyperkeratosis [3]. Moreover, the footprints reflecting a large amount of information regarding

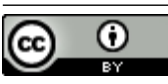

This work is licensed under a Creative Commons Attribution International 4.0 License.

BECB 2021, August 13-15, 2021, Nanchang, China

(C) 2021 Copyright held by the owner/author(s)

ACM ISBN 978-1-4503-8411-7/21/08.

https://doi.org/10.1145/3502060.3502148 people's health status and gestures can also provide valuable information for fall prediction, the diagnosis of many diseases and rehabilitation therapy.

There are in general three kinds of techniques for obtaining footprints, i.e. stamp mark method, optical scanning method and force-sensitive piezoresistive film method [4]. The stamp mark method is the simplest strategy, with which the footprints can be obtained by observing the marks left on the deformed materials, such as sand, mud and rubber [5]. However, this method can only obtain a static image of the footprint and cannot achieve an accurate map of pressure distribution. For the optical scanning method, an elastic pad is placed rubber on the glass, and the glass is exposed to the light sources at both sides. The pressure of the foot on the elastic pad causes the deformation of the elastic medium, which affects the light reflected by the glass and the intensity of light is usually proportional to the pressure [6]. However, the accuracy of this method is limited and it requires a space under the foot to palace the mechanic devices. In recent years, the development of piezoresistive films makes it possible to accurately obtain the plantar pressure distribution on the piezoresistive film [7]. The piezoresistive films has many force-sensitive resistors whose resistances can respond to the variation of the applied force in microseconds [8]. By appropriately designing an electric circuit, the varying resistance can be transferred to the voltage signal, then through analog-digital converter, the applied pressure on the force-sensitive points can be obtained [9]. Thanks to the progress of circuit process manufacturing, a piezoresistive film with thickness under the millimeter level can be fabricated. Owing to the lightweight, small size and high accuracy property of the piezoresistive film, it has been considered as the best candidate used to obtaining the plantar pressure distribution [10].

In recent decades, numerous researchers have dedicated to investigate the relationship between planter pressure distribution and both kinematics [11] and electromyography [12]. The plantar pressure analysis in conjunction with lower limb biomechanics, electromyography and kinematics are also introduced to find the relation between foot posture and lower limb biomechanics [13]. A study found an abnormal plantar pressure distribution in diabetic patients that compared to the normal people, the load of the heel and midfoot is higher during walking [14]. These studies involve the analysis of footprints, which normally requires many manual works on dealing with the data of footprint. Therefore, it would be a time-consuming process to analysis the footprints with a large amount of data [15], [16]. To tackle this problem, in this study, a statistical method is proposed to distinguish between left footprint and right footprint and to obtain the directions of the footprints 

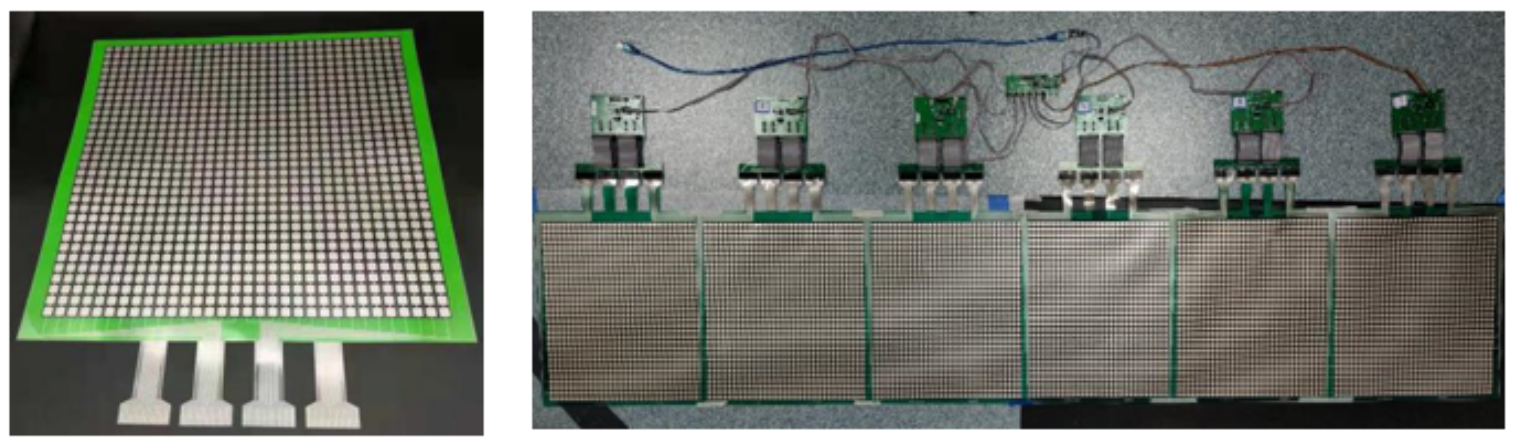

Figure 1: (a) One piece of piezoresistive film; (b) Six pieces of the piezoresistive films and data acquisition devices

and the step length, which are the main parameters for the analysis of footprints. Moreover, the obtained parameters can also be to reconstruct the walking map, which can significantly reduce the storage of the footprints data.

\section{THE DEVELOPMENT OF THE HARDWARE SYSTEM}

The piezoresistive film (Rouxi, RX-M3232L), as shown in Figure 1 (a), has 1024 force-sensitive points, which is made by transferring forcesensitive nano-ink, silver paste and other materials to the flexible film substrate (PET or PI), then drying and curing. Six pieces of the piezoresistive films were adopted to extend the force-sensitive area up to $\left(2400 \mathrm{~mm}^{*} 400 \mathrm{~mm}\right)$, as shown in Figure 1 (b). Each force sensitive point can be located by selecting a channel in the row and a channel in the column. The resistance of the force-sensitive points with respect to the applied force is provided in Figure 2, which shows that the resistance of the force-sensitive points decreases with the increase of pressure. A voltage divider circuit is adopted to measure the voltage of the measurement point. The increase of the applied pressure on the force-sensitive point leads to the decrease of the resistance and the voltage of the measurement point. Subsequently, digital-to-analog converter is utilized to convert the measured voltage into digital data, then the data are transferred to the computer by using the designed data acquisition devices shown in the Figure $1(b)$.

\section{A STATISTICAL METHOD FOR GAIT ANALYSIS}

In this section, a statistical method for footprint analysis is proposed to distinguish between left footprint and right footprint and to obtain the direction of the footprints and the step length, which are the most parameters for footprints analysis.

To obtain the data of footprints, a software that can receive the data from the designed signal acquisition devices through the serial port and visualize the footprint was developed. The visual image of six footprints on the piezoresistive films are represented in Figure 3, in which a small square represents a force-sensitive unit in piezoresistive films and different levels of the pressure values are presented with different colors. The relative position of the force-sensitive units can be calculated using the matrix (32*192). The visual image is updated at about 12 frames per second, which

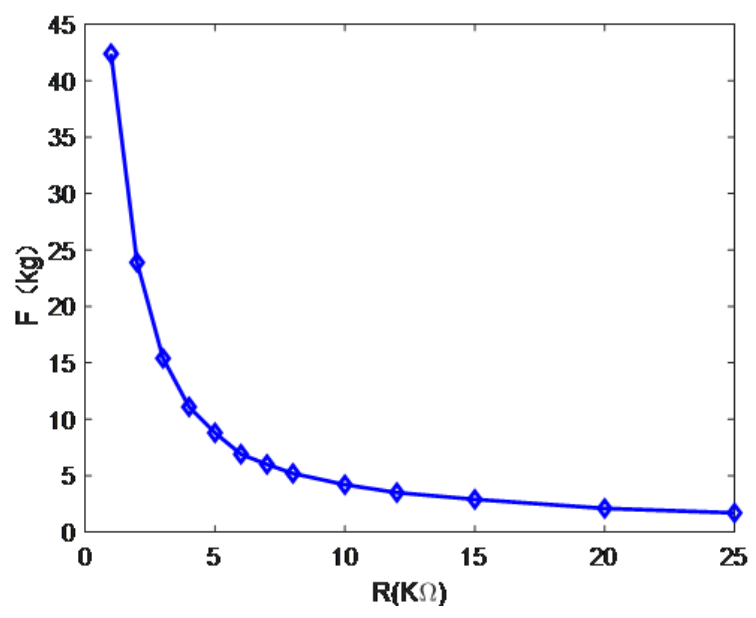

Figure 2: The experimental data of the resistance of the force-sensitive points with respect to the applied force

allows capturing the dynamic changes of the footprint at wide range of the walking speed. Considering that multiple images may be captured when walking on the piezoresistive film, the frame with the largest area is used for further analysis. Due to a variety of disturbances in the hardware of the signal acquisition device, a few small activated areas may show on the image, which may deteriorate the visual image. Thus, a threshold method is adopted to filter out the unexpected noise.

To begin with, the adjacency matrixes of the footprints are obtained, as presented in Figure 4, then the angle R between the lower edge of the adjacency matrixes and the $\mathrm{x}$-axis are calculated to rotate the footprints to be parallel to the $\mathrm{x}$-axis. The vertical projections of the adjacency matrixes are computed to locate the position of the hell and the ball of the foot, and the variations of the vertical projection value with respect to the column number are presented in Figure 5. From which, three peaks can be clearly seem, where the two largest peaks are caused by the pressure of the ball of foot and heel and the smallest peak is caused by toes. Due to the dynamic change of the footprint during the walking, it is inaccurate to determine the position of the heel and the ball of foot by comparing 


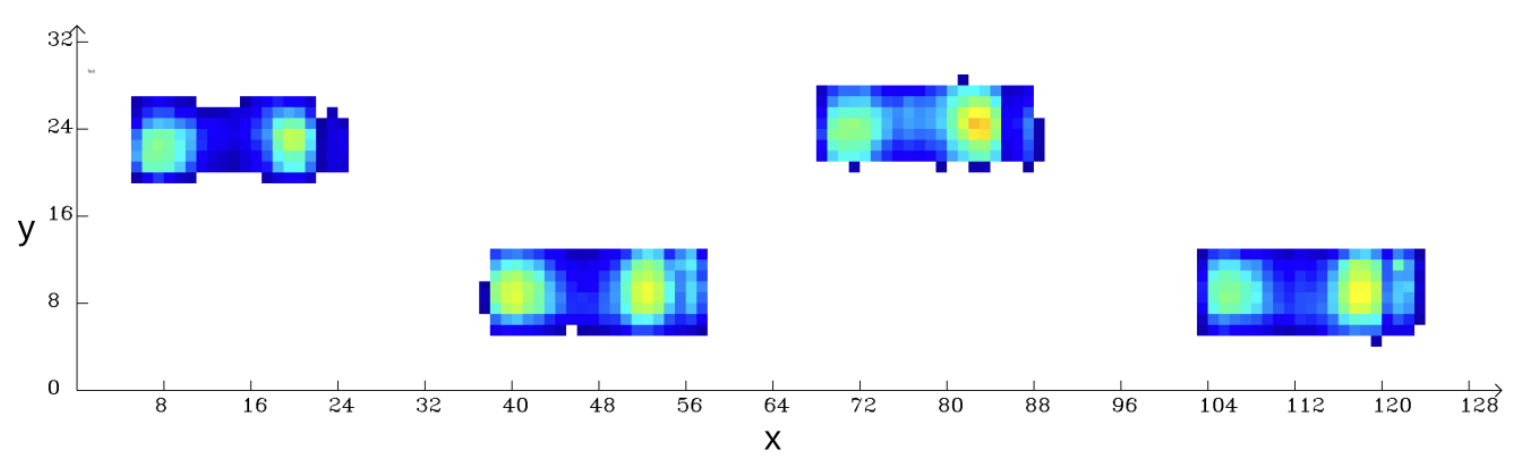

Figure 3: The image of six footprints on the piezoresistive films

the value of the two largest peaks. Therefore, the sum value in the first three columns and the sum values in the last three columns are compared to distinguish the area of the heel and ball of foot It is assumed that the heel area is near to the three columns with the greater value, while the area of ball of foot is near to the three columns with the smaller columns.

The left and right footprints, as shown in Figure 4 (a) and (b), can be identified based on a significant difference on their geometric shape. As the right footprint shown in Figure 4 (b), it can be recognized that if the position of the medial arch is on the upside and the lateral arch is on the downside, this footprint is identified as the right one. On the contrary, if the position of the medial arch is on the downside and the lateral arch is on the upside, this footprint is identified as the left one. Therefore, the geometric feature of the arches is used to distinguish the left and right footprints. Firstly, it is defined that the arch area is formed by the column lines of the two largest peaks and the long sides of the adjacency matrixes. Subsequently, two parts (the upper half and the bottom half part) are extracted from the arch area, as indicated in Figure 4 (b). The method of computing the area of the upper half and the bottom half is described using the pseudo code as shown in Figure 6. The areas of the upper half the bottom half are compared, and it is assumed that the medial arch is in the part with larger area and the lateral arch is in the part with smaller area.

Prior to obtain the center of gravity coordinates of the heel and the ball of the foot, the areas of the heel and ball of foot in the adjacency matrix are divided by the column line of the minimum value between the two largest peak value. The coordinates of the center of the heel in the matrix are denoted as $\left(x_{h}, y_{h}\right)$, which are calculated as :

$$
\begin{gathered}
x_{h}=\frac{\sum_{(x, y) \in \Omega_{h}} x \cdot p(x, y)}{\sum_{(x, y) \in \Omega_{h}} p(x, y)} \\
y_{h}=\frac{\sum_{(x, y) \in \Omega} y \cdot p(x, y)}{\sum_{(x, y) \in \Omega} p(x, y)}
\end{gathered}
$$

where $\Omega_{h}$ represents the area of heel. The coordinates of the center of the ball of foot in the matrix are denoted as $\left(x_{h}, y_{h}\right)$, which are calculated as :

$$
x_{b}=\frac{\sum_{(x, y) \in \Omega_{b}} x \cdot p(x, y)}{\sum_{(x, y) \in \Omega_{b}} p(x, y)}
$$

$$
y_{b}=\frac{\sum_{(x, y) \in \Omega_{b}} y \cdot p(x, y)}{\sum_{(x, y) \in \Omega_{b}} p(x, y)}
$$

where $\Omega_{b}$ represents the area of the ball of foot. Based on the coordinates of the centers, the direction of the footprints can be determined. The step length of the two consecutive footprints is calculated by computing the distance of the center of two consecutive adjacency matrixes, which can present as:

$$
L_{s}=\sqrt{\left(x_{c+1}-x_{c}\right)^{2}+\left(y_{c+1}-y_{c}\right)^{2}}
$$

Where $L_{s}$ is dented as the step length of two consecutive footprints. $x_{c+1}$ and $x_{c}$ denotes the horizontal coordinates of the centers of two consecutive adjacency matrixes, while $y_{c+1}$ and $y_{c}$ denotes the longitudinal coordinates of the centers of two consecutive adjacency matrixes.

\section{EXPERIMENTAL RESULT}

In this section, a series of experiments are conducted to obtain the accuracy of the proposed method used for different foot sizes and different walking speeds. In the experiments, three feet with different foot lengths, A $(270 \mathrm{~mm}), B(265 \mathrm{~mm})$ and C $(285 \mathrm{~mm})$ are considered and the walking speeds are classified as: slow speed $(0.5 \sim 1.1 \mathrm{~m} / \mathrm{s})$, normal walking speed $(1.1 \sim 1.5 \mathrm{~m} / \mathrm{s})$, quick walking speed $(1.5 \sim 1.8 \mathrm{~m} / \mathrm{s})$ and the slow running speed $(1.8 \sim 2.1 \mathrm{~m} / \mathrm{s})$. For each subject in each walking speed, 114 effective footprints are collected to calculate the accuracy through manual work. The errors may occur due to the following reasons: a wrong distinction between the right and left feet, a wrong distinction between the heel and the ball of foot and miscalculating the center of the heel and the ball of foot. The accuracy of proposed strategy with respect to different foot sizes and different walking speeds are provided in Table 1. The result shows the accuracy of the proposed method is independent with the foot lengths, but is dependent with the walking speed. The accuracy of the proposed method is decreased with the increase of the walking speed, as in a fast-walking speed the image of the planter pressure distribution may be incomplete.

\section{CONCLUSION}

In this study, six pieces of the piezoresistive films were adopted to extend the force-sensitive area up to $\left(2400 \mathrm{~mm}^{*} 400 \mathrm{~mm}\right)$ with $1024^{*} 6$ force-sensitive units, and data acquisition devices were developed 


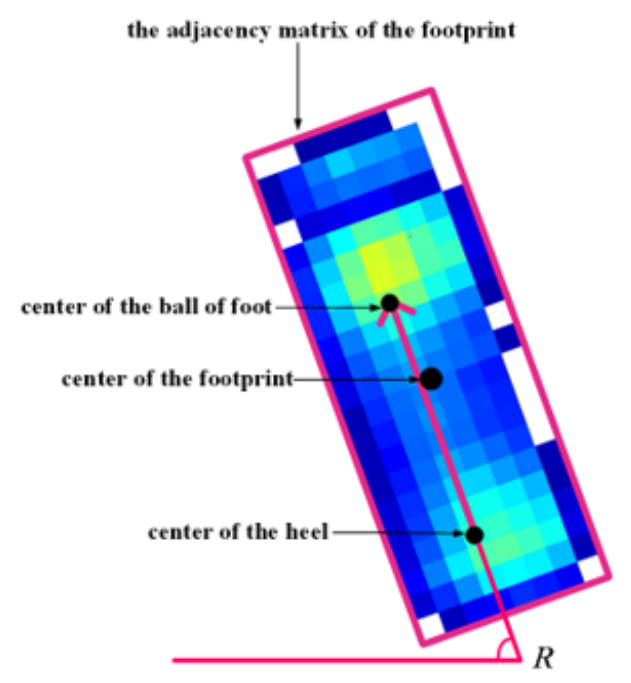

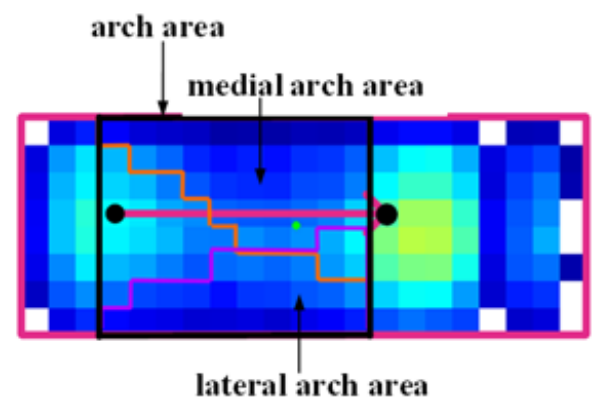

lateral arch area

Figure 4: (a) The planter pressure distribution of the right foot; (b) The planter pressure distribution of the left foot

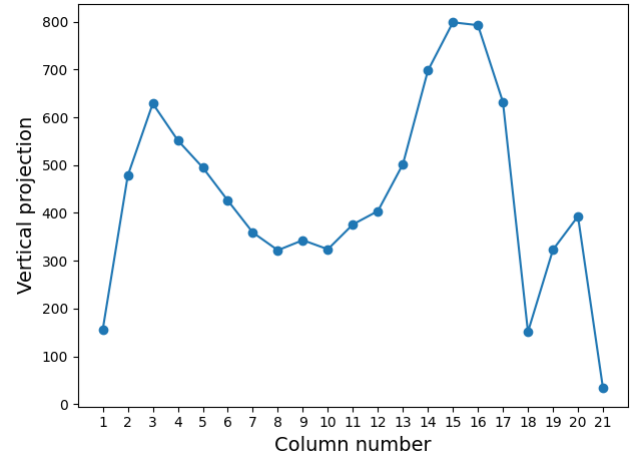

Figure 5: The vertical projection of the adjacency matrix

Table 1: The accuracy of proposed strategy with respect to different foot sizes and different walking speeds

\begin{tabular}{llll}
\hline Walking speeds & $\mathrm{A}(270 \mathrm{~mm})$ & $\mathrm{B}(265 \mathrm{~mm})$ & $\mathrm{C}(285 \mathrm{~mm})$ \\
\hline $0.5 \sim 1.1 \mathrm{~m} / \mathrm{s}$ & 1 & 1 & 1 \\
$0.5 \sim 1.5 \mathrm{~m} / \mathrm{s}$ & 0.83 & 0.77 & 0.73 \\
$1.5 \sim 1.8 \mathrm{~m} / \mathrm{s}$ & 0.75 & 0.68 & 0.625 \\
$1.8 \sim 2.1 \mathrm{~m} / \mathrm{s}$ & 0.61 & 0.55 & 0.50 \\
\hline
\end{tabular}

to obtain the plantar pressure distribution by measuring the voltage changes caused by the applied pressure on the piezoresistive films. A software was developed to receive the data through the serial port and visualize the footprints. A statistical method was proposed to distinguish between left footprint and right footprint and to obtain the direction of the footprints and the step length, which can be used to replace the manual work of obtaining the main parameters of footprint data. Eventually, a series of experiments were conducted to verify the effectiveness of the proposed method and the result shows that the accuracy of the proposed method is independent with the foot lengths, but is dependent with the walking speed and the accuracy is up to $100 \%$ when the walking speed is lower than $1.1 \mathrm{~m} / \mathrm{s}$.

\section{ACKNOWLEDGMENTS}

This work was supported in part by the Guangdong Province Science and Technology Program Grant (2018A050501010), the Shenzhen Governm ental Science and Technology for Basic Research Program Grant (JCYJ20180507182446643), and the Shenzhen Science and Technology for International Cooperation Research Program Grant (GJHZ20190821155201661).

\section{REFERENCES}

[1] Pauk J, Griskevicius J, Daunoraviciene K, et al. 2010. Analysis of the plantar pressure distribution in children with foot deformities. Acta of Bioengineering \& Biomechanics, 12(1).

[2] Callewaert B, Deschamps K, Matricali G, et al. 2014. Classification of Forefoot Plantar Pressure Distribution in Persons with Diabetes: A Novel Perspective for the Mechanical Management of Diabetic Foot? Gait \& Posture 39(11) S90-S91.

[3] Goulermas J Y, Findlow A H, Nester C J, et al. 2005. Automated design of robust discriminant analysis classifier for foot pressure lesions using kinematic data. IEEE Transactions on Biomedical Engineering, 2005, 52(9):1549-1562.

[4] Bautista J, SLC Cárdenas, Zavala A H, et al. 2018. Review on plantar data analysis for disease diagnosis. Biocybernetics and Biomedical Engineering, 2018, 342-361.

[5] Razak A A, Zayegh A, Begg R K, et al. 2012. Foot Plantar Pressure Measurement System: A Review. Sensors, 12(7):9884-9912.

[6] Panjan A, Sarabon N. 2010. Review of Methods for the Evaluation of Human Body Balance. Sport Science Review, 19(5-6):131-163.

[7] Chen B, Wang X, Huang Y, et al. 2015. A foot-wearable interface for locomotion mode recognition based on discrete contact force distribution. Mechatronics, 2015.

[8] Kwon D, Lee T I, Shim J, et al. 2016. Highly sensitive, flexible, and wearable pressure sensor based on a giant piezocapacitive effect of three-dimensional microporous elastomeric dielectric layer. ACS Applied Materials \& Interfaces, 2015: 16922-16931.

[9] Mei H, Wang R, Wang Z, et al. 2015. A flexible pressure-sensitive array based on soft substrate. Sensors and Actuators A: Physical, 2015.

[10] Mannsfeld S, Tee C K, Stoltenberg R M, et al. 2010. Highly sensitive flexible pressure sensors with microstructured rubber dielectric layers. Nature Materials, $9(10): 859-864$. 


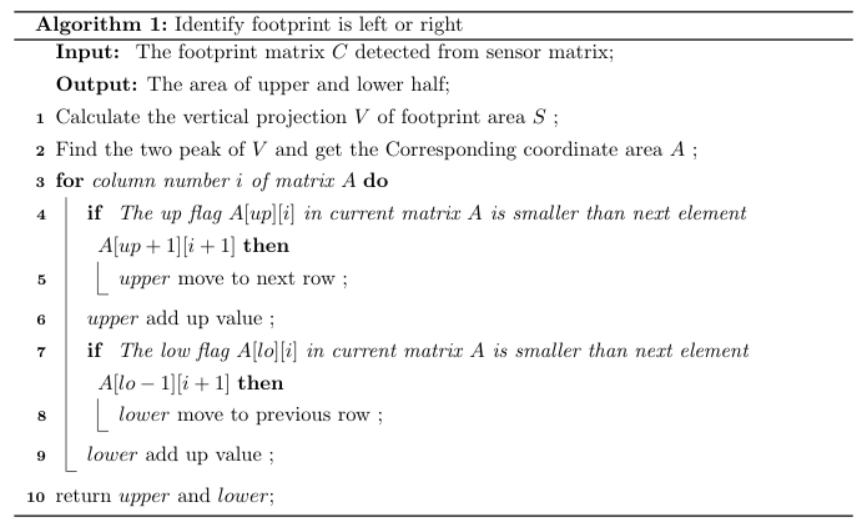

Figure 6: The pseudo code of calculation of the area of the upper and lower part of the arch of the foot.

[11] Buldt A K, Murley G S, Butterworth P, et al. 2013. The relationship between foot posture and lower limb kinematics during walking: A systematic review. Gait \& Posture, 38(3): 363-372.

[12] Murley G S, Landorf K B, Menz H B, et al. 2009. Effect of foot posture, foot orthoses and footwear on lower limb muscle activity during walking and running: a systematic review. Gait \& Posture, 29(2): 172-187.

[13] Landorf K B, Keenan A M. 2000. Efficacy of foot orthoses. What does the literature tell us? Journal of the American Podiatric Medical Association, 90(3): 149-158.
[14] Bacarin T A, Sacco I C N, Hennig E M. 2009. Plantar pressure distribution patterns during gait in diabetic neuropathy patients with a history of foot ulcers. Clinics, 64(2): $113-120$.

[15] Igor, Kononenko, 2001. Machine learning for medical diagnosis: history, state of the art and perspective - ScienceDirect. Artificial Intelligence in Medicine, 23(1):89-109.

[16] Polat K, Salih Güne, 2007. An expert system approach based on principal component analysis and adaptive neuro-fuzzy inference system to diagnosis of diabetes disease. Digital Signal Processing, 17(4):702-710. 